Progress in Quantum Field Theory and String Theory International Journal of Modern Physics: Conference Series

Vol. 21 (2013) 195-196

(C) World Scientific Publishing Company

DOI: 10.1142/S201019451300977X

\title{
LOCALIZATION AND KNOTS
}

\author{
AKINORI TANAKA \\ Department of Physics, Graduate School of Science, \\ Osaka University, Toyonaka, Osaka 560-0043, Japan \\ akinori@hetmail.phys.sci.osaka-u.ac.jp
}

\begin{abstract}
We study the 1/2 BPS condition of Wilson loop on squashed three-sphere, and find that the solution becomes torus knot or unknot. We also calculate the expectation value of $1 / 2$ BPS Wilson loops by using localization technique with the gauge group $U(2)$ and Wilson loop as fundamental representation. And it completely matches with known results.
\end{abstract}

Keywords: Localization; knots.

\section{Introduction}

As well known, the expectation values of fundamental-representation Wilson loops with pure $S U(2)$ Chern-Simons theory on the three-sphere turn to be celebrated Jones polynomials. ${ }^{1}$ On the other hand, one can perform exact calculation of $\mathcal{N}=2$ SUSY Chern-Simons theory's path integral by using localization technique. We can check the consistency between these two exact results through the knot theory.

\section{1/2 BPS Condition}

We use localization procedure on squashed three-sphere. ${ }^{2}$ The metric is

$$
d s^{2}=f(\theta)^{2} d \theta^{2}+l^{2} \cos ^{2} \theta d \phi^{2}+\tilde{l}^{2} \sin ^{2} \theta d \chi^{2}, \quad f(\theta)=\sqrt{l^{2} \sin ^{2} \theta+\tilde{l}^{2} \cos ^{2} \theta} .
$$

We calculate following supersymmetric Wilson loop

$$
W_{S}=\operatorname{Tr}_{R} \mathcal{P} \exp \left(\oint_{C} d \tau\left(i A_{\mu} \dot{x}^{\mu}+\sigma|\dot{x}|\right)\right)
$$

weighted by $\exp \left(i k S_{S C S}\right)$ where

$$
S_{S C S}=\int d^{3} x \sqrt{g} \operatorname{Tr}\left[\frac{1}{\sqrt{g}} \epsilon^{\mu \nu \lambda}\left(A_{\mu} \partial_{\nu} A_{\lambda}-\frac{2 i}{3} A_{\mu} A_{\nu} A_{\lambda}\right)-\bar{\lambda} \lambda+2 D \sigma\right] .
$$

In order to apply localization technique, Wilson loop contour $x^{\mu}(t)=(\theta(t)$, $\phi(t), \chi(t))$ must satisfy following $1 / 2$ BPS condition ${ }^{3}$

$$
\dot{x}^{\mu} \frac{\partial}{\partial x^{\mu}}=\left\{\begin{array}{ll}
\frac{1}{l} \frac{\partial}{\partial \phi}-\frac{1}{\tilde{l}} \frac{\partial}{\partial \chi} & \left(\theta \neq 0, \frac{\pi}{2}\right) \\
\frac{1}{l} \frac{\partial}{\partial \phi} & (\theta=0) \\
-\frac{1}{\tilde{l}} \frac{\partial}{\partial \chi} & \left(\theta=\frac{\pi}{2}\right)
\end{array} .\right.
$$




\section{Integral Curves and Results of $U(2)$ Gauge Theory}

We summarize the results on the following table.

\begin{tabular}{|c|c|c|c|}
\hline & $\theta=0$ & $\theta \neq 0, \pi / 2$ & $\theta=\pi / 2$ \\
\hline $\begin{array}{l}1 / 2 \text { BPS } \\
\text { Wilson loop } \\
\text { on } S^{3}\end{array}$ & & & $\uparrow$ \\
\hline $\begin{array}{c}1 / 2 \text { BPS } \\
\text { Wilson loop } \\
\text { on } T^{2} \subset S^{3}\end{array}$ & $\chi$ & $\chi$ & $\chi$ \\
\hline$\frac{\left\langle W_{S}\right\rangle}{\langle 1\rangle}$ & $e^{-\frac{l}{l} \frac{2 \pi i}{k}} \frac{q-q^{-1}}{q^{1 / 2}-q^{-1 / 2}}$ & $\begin{array}{c}e^{-l \tilde{l} \frac{2 \pi i}{k}} \frac{\frac{1}{2}(l-1)(\tilde{l}-1)^{1-q^{2}}}{} \times \\
\left(1+q^{l+\tilde{l}}-q^{l+1}-q^{\tilde{I}+1}\right) \frac{q-q^{-1}}{q^{1 / 2}-q^{-1 / 2}}\end{array}$ & $e^{-\frac{\tilde{l}}{l} \frac{2 \pi i}{k}} \frac{q-q^{-1}}{q^{1 / 2}-q^{-1 / 2}}$ \\
\hline
\end{tabular}

For simplicity, we draw $(\theta \neq 0, \pi / 2)$ picture by substituting $(l=2, \tilde{l}=3)$. Here we performed the calculation with gauge group $U(2)$ and Wilson loop's representation $R$ as fundamental-representation. And we denote $e^{\frac{2 \pi i}{k}}$ as $q$. These results correspond to the level $(k-2)$ pure Chern-Simons theory.

\section{Remark}

Polynomials on the table completely match with $(l, \tilde{l})$-torus knot Jones polynomial and unknot Jones polynomial respectively up to overall phase factor. This phase comes from the point-splitting regularization of Wilson loop. It is also possible to calculate the correlation function of many $1 / 2$ BPS Wilson loops by inserting $1 / 2$ BPS Wilson loops into the integrand. ${ }^{3}$

\section{References}

1. E. Witten, "Quantum Field Theory and the Jones Polynomial," Commun. Math. Phys. 121 (1989) 351.

2. N. Hama, K. Hosomichi, and S. Lee, "SUSY Gauge Theories on Squashed ThreeSpheres," JHEP 05 (2011) 014, arXiv:1102.4716 [hep-th].

3. A. Tanaka, "Comments on knotted 1/2 BPS Wilson loops," arXiv:1204.5975 [hep-th]. 